\title{
MECHANISM OF SUPERCRITICAL FLUID EXTRACTION OF COAL
}

\author{
TADAfumi ADSCHIRI, Shinji ABE AND Kunio ARAI \\ Department of Biochemistry and Chemical Engineering, Tohoku University, \\ Sendai 980
}

Key Words: Energy, Extraction, Coal, Supercritical Fluid, Mechanism, Liquefaction

\begin{abstract}
This paper describes the mechanism of supercritical toluene (or toluene-tetralin mixture) extraction of coal. The effects of solvent power and solvent residence time in the cell on conversion and liquid yield were examined by using a two-stage extraction method: extraction at various conditions followed by high-solvent power extraction. The effect of tetralin addition on supercritical toluene extraction was also investigated by this method. Furthermore, in situ ESR measurement was conducted to evaluate the variation of radical concentration during supercritical fluid extraction. From these detailed analyses the effects of the solvent power of supercritical fluid on the reactions during extraction, namely on decomposition and polymerization of the primary products from coal decomposition, and on stabilization of the radicals through hydrogen transfer reaction were evaluated.
\end{abstract}

\section{Introduction}

Supercritical fluid has higher diffusivity and lower viscosity than a liquid, and its solvent power can be controlled by temperature and/or pressure. Supercritical fluid extraction of coal using this specific feature is a promising coal conversion method which may control the product distribution.

Some researchers ${ }^{1-4}$ have investigated the effects of temperature, pressure, type of solvent and type of coal on conversion and liquid yield. However, the relation between extraction conditions and product distribution has not been fully elucidated yet.

One reason for the difficulty of elucidating the mechanism of supercritical fluid extraction is the complicated effects of temperature and pressure on extraction performance. Both temperature and pressure affect the fluid's solvent power, as well as the thermal decomposition of coal itself. Furthermore, secondary reactions (decomposition, polymerization and stabilization by hydrogen transfer) of the primary products of coal decomposition are not independent of solvent power. For example, decomposition, polymerization and stabilization of the primary products may be inhibited by their rapid extraction. The authors consider that clarification of the effect of the supercritical fluid's solvent power on the secondary reactions of the primary products is essential to elucidating the mechanism of the supercritical fluid extraction of coal.

The objectives of this study are to examine in detail the effect of the supercritical fluid's solvent power on the secondary reactions of the primary products and

* Received May 7, 1991. Correspondence concerning this article should be addressed to T. Adschiri. to elucidate the mechanism of supercritical toluene (and toluene-tetralin mixture) extraction of coal.

\section{Experimental}

\subsection{Supercritical fluid extraction with semi-batch type reactor}

Taiheiyo coal was used as a sample coal. Proximate and ultimate analyses of the coal are shown in Table 1. Particle size of coal was $0.29-0.50 \mathrm{~mm}$.

A schematic diagram of the reaction system used is shown in Fig. 1. A high-pressure stainless steel tube $(0.8 \mathrm{~cm}$ id, $6.0 \mathrm{~cm} \mathrm{l})$ was employed as an extraction cell. About $8 \mathrm{~g}$ of dried coal was loaded in the middle

Table 1. Proximate and ultimate analyses of Taiheiyo coal

\begin{tabular}{cccccccccc}
\hline $\begin{array}{c}\text { Proximate Analysis } \\
{[\mathrm{wt} \% \mathrm{db}]}\end{array}$ & \multicolumn{6}{c}{$\begin{array}{c}\text { Ultimate Analysis } \\
{[\mathrm{wt} \% \mathrm{daf}]}\end{array}$} \\
\cline { 1 - 2 } & Ash $\begin{array}{c}\text { Volatile Fixed } \\
\text { matter carbon }\end{array}$ & & $\mathrm{C}$ & $\mathrm{H}$ & $\mathrm{N}$ & $\mathrm{S}$ & $\mathrm{O}$ (diff.) & $\mathrm{H} / \mathrm{C}[-]$ \\
\hline 14.2 & 45.0 & 40.8 & 77.5 & 6.4 & 1.2 & 0.3 & 14.6 & 0.99 \\
\hline
\end{tabular}

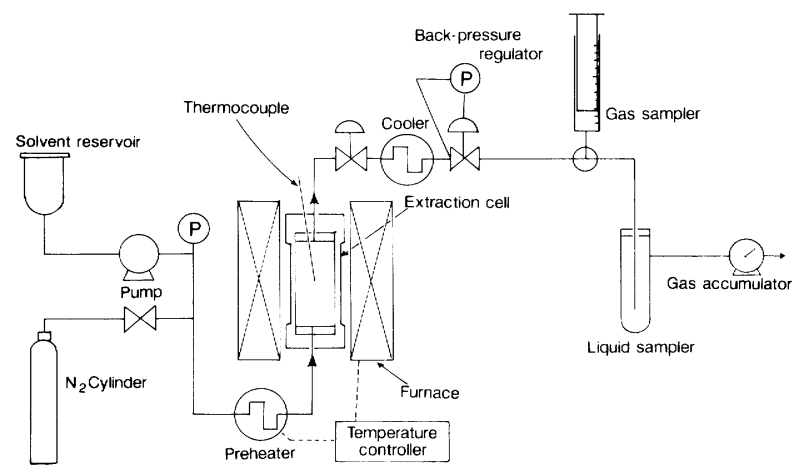

Fig. 1. Experimental apparatus 
part of the cell. On both sides of the coal bed, glass wool was placed to avoid elution of coal from the cell.

In this study, to determine the effect of the supercritical fluid's solvent power on the reactions during extraction, a two-stage extraction method was employed. In the first stage, supercritical fluid extraction was conducted at various conditions and in the second stage the residual products in the coal bed were recovered by high-solvent power extraction. Since the extraction condition could be kept the same in each series of experiments by this procedure, the effects of solvent power on the product distribution of the first stage could be examined.

Solvent (toluene or toluene-tetralin (30\%) mixture) was introduced into the cell at a feed rate of $2.5 \mathrm{Nml} / \mathrm{min}$ and the system was raised to reaction pressure $(0.1-20 \mathrm{MPa})$. In some runs, nitrogen was fed at $0.5 \mathrm{Nl} / \mathrm{min}$ rather than the solvent in order to elucidate the effect of pressure on the reaction itself. Then the cell was heated to $653 \mathrm{~K}$ at a heating rate of $10 \mathrm{~K} / \mathrm{min}$ and kept at this temperature for 1.5 hour. Coal decomposition and secondary reactions of the primary products took place during this procedure. A portion of the products was continuously withdrawn from the cell along with solvent. Then toluene or toluene-tetralin mixture at a pressure of $20 \mathrm{MPa}$ was fed into the cell for 1.5 hour to recover the residual products in coal particles.

The weight of the residual coal was measured and the conversion of coal was evaluated by the weight change of the sample coal. The amount of gas produced during the extraction was measured by a gas accumulator and was found to be negligibly small. The extract was collected with the solvent in a sampling vessel. The solvent was distilled at $403 \mathrm{~K}$ under reduced pressure and the products obtained were dried at $403 \mathrm{~K}$ under vacuum. During this treatment, light components contained in the extract were evaporated. Volatile matter was defined as the fraction volatilized during the drying under vacuum. The rest of the extract was defined as liquid products. Thus the conversion of coal, $X$, liquid yield, $Y_{L}$, and yield of volatile matter, $Y_{V}$, are given by equations 1 to 3 respectively.

$$
\begin{gathered}
X=\frac{W_{S}-W_{R}}{W_{S}} \times 100 \\
Y_{L}=\frac{W_{L}}{W_{S}} \times 100 \\
Y_{V}=X-Y_{L}
\end{gathered}
$$

where $W_{S}$ [g-daf ] is weight of loaded coal, $W_{R}$ [g-daf ] is weight of residual coal, and $W_{L}$ [g-daf] is weight of liquid product.

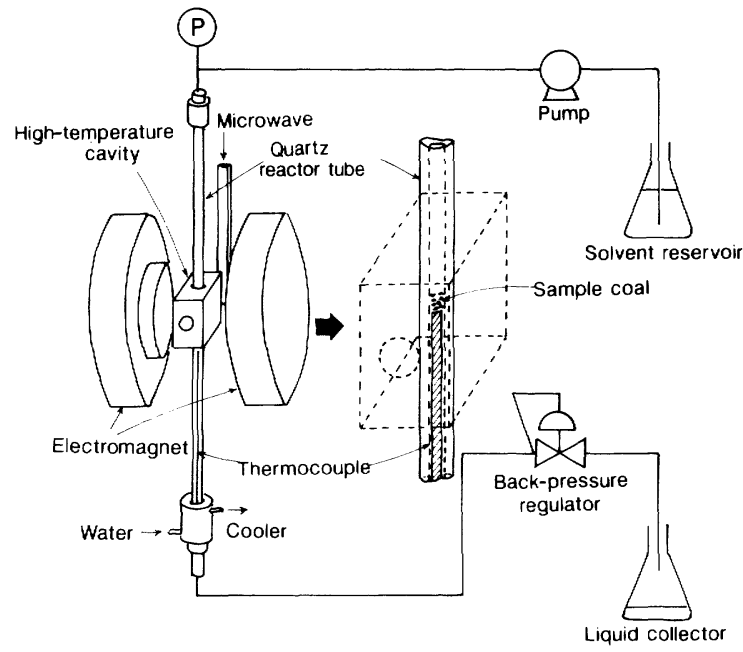

Fig. 2. In situ ESR apparatus

\subsection{ESR measurement}

A schematic diagram of the apparatus employed in ESR measurement is shown in Fig. 2. A quartz tube ( $2 \mathrm{~mm}$ id.) was used as a reactor. About $40 \mathrm{mg}$ of sample coal with particle size ranging from 0.29 to $0.50 \mathrm{~mm}$ was loaded in the reactor. A thermocouple inserted through a tube was located just below the sample. Solvent was fed into the reactor at $0.5 \mathrm{Nml} / \mathrm{min}$. ESR apparatus used was a model JEOL FE3X equipped with a high-temperature cavity (Micro Device Ltd.). Modulation of $100 \mathrm{kHz}$ and a nominal operating frequency of $9.5 \mathrm{GHz}$ were chosen for the sound evaluation of ESR spectra, based on the results of a preliminary experiment.

The reactor was heated to $653 \mathrm{~K}$ at a heating rate of $10 \mathrm{~K} / \mathrm{min}$. ESR spectra, $I(T)$, were obtained at intervals of about $3 \mathrm{~min}(30 \mathrm{~K})$ during the heating process. Then the temperature was kept at $653 \mathrm{~K}$ for about $30 \mathrm{~min}$, during which the reaction was completed. To evaluate the effect of temperature on the intensity of ESR spectra, the spectra of residual coal, $I_{r}(T)$, were also measured. After cooling to room temperature, the reactor with the residual coal was heated again at the same heating rate and ESR spectra during this procedure, $I_{r}(T)$, were measured. Thus the relative intensity, $I(T) / I_{\mathrm{r}}(T)$, was evaluated to eliminate the effect of temperature on the intensity of the ESR spectrum. Furthermore, to compensate for the effects of solvent and amount of loaded coal on the intensity of the ESR spectrum, the relative intensity was normalized by that obtained at $298 \mathrm{~K}$, as $I(T) / I_{r}(T) /\left\{I(298) / I_{r}(298)\right\}$.

\section{Results and Discussion}

\subsection{Supercritical toluene extraction}

The relative intensity of ESR spectra obtained are shown in Fig. 3. Under an atmosphere of $0.1 \mathrm{MPa}$ toluene the intensity of the ESR spectra increased 


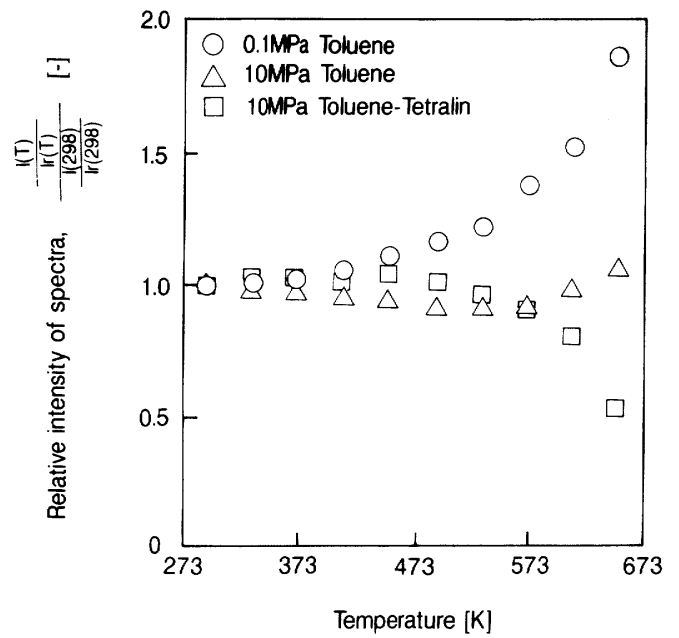

Fig. 3. Intensity of ESR spectra during supercritical fluid extraction

with increasing temperature. Generally, the intensity of the ESR spectrum was closely related to the amount of radicals in the cell. Thus the increase in intensity may be attributed to the increase in amount of radicals with temperature due to coal decomposition. However, during toluene extraction at $10 \mathrm{MPa}$, the variation of intensity with temperature was not significant. The difference between these curves may be due to the extraction of the unstable primary products of coal decomposition before their decomposition in the cell.

To examine whether thermally unstable species exist in the extract, the effect of solvent residence time on extract composition was investigated by changing the feed rate of solvent. As shown in Fig. 4, the volatile yield increased with increasing residence time. The liquid products (residence time, $83 \mathrm{sec}$ ), once recovered, were fed again to the bed of residual coals at the same temperature, pressure and feed rate. The result is plotted in Fig. 4, assuming that the residence time was doubled (residence time, $166 \mathrm{sec}$ ). It was found that a portion of the liquid products turned into volatile species. These results suggest that a portion of the primary liquid products of thermal decomposition of coal decompose into volatiles in a reaction time of around $2 \mathrm{~min}$. However, conversion changed little over this range of residence time. This suggests that the effect of residence time on the polymerization of the unstable species is negligible under the present experimental conditions.

To examine the catalytic effect of ash in coal on the decomposition of liquid, the recovered extract (residence time, $83 \mathrm{sec}$ ) was further fed to a glass beads bed $(2 \mathrm{~mm} \phi)$ instead of the coal bed, at $653 \mathrm{~K}$ and $20 \mathrm{MPa}$ and at a feed rate of $0.5 \mathrm{Nml} / \mathrm{min}$. As a result, the liquid yield decreased, while volatile yield increased. As shown in Fig. 4 (symbols $\odot$, $\square$ ), these

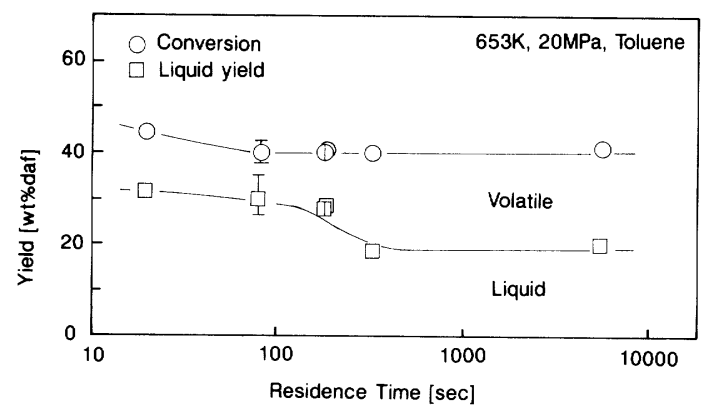

Fig. 4. Effect of residence time on conversion and liquid yield through supercritical toluene extraction

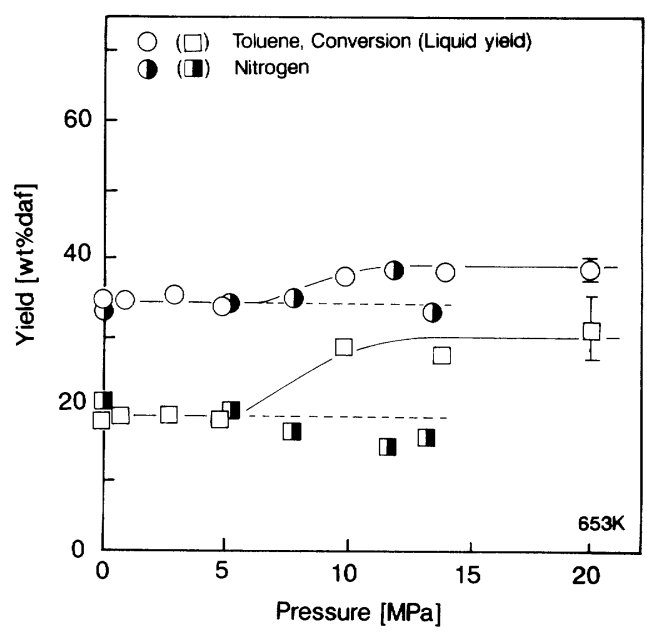

Fig. 5. Effect of pressure on conversion and liquid yield through supercritical toluene extraction

yields were found to fall on the same curve. Thus it is concluded that the decomposition of liquid is a noncatalytic reaction.

Prior to the experiments for examining the effect of the supercritical toluene's solvent power on product distribution, the effect of pressure on the coal decomposition itself was investigated under a nitrogen atmosphere. After flowing nitrogen for 1.5 hour, the products were recovered by supercritical toluene at $20 \mathrm{MPa}$ and $653 \mathrm{~K}$; the results are shown in Fig. 5. Neither the conversion of coal nor liquid yield was largely affected by the nitrogen pressure. This suggests that the effect of pressure on the decomposition of coal itself is not significant under the present experimental condition.

The effect of pressure during supercritical toluene extraction on the conversion and the liquid yield is also shown in Fig. 5. Since the experiments were conducted under the condition where residence time of solvent in the reactor was less than $2 \mathrm{~min}$, the products which had been once dissolved in the supercritical toluene were supposed to be removed from the cell before further reactions could occur, as suggested in Fig. 4. The products which had remained in coal particles were considered to be 
decomposed or polymerized.

Figure 5 shows that liquid yield increased with increasing pressure, while volatile yield decreased. This result can be explained by taking into account the change of solvent power of supercritical fluid with pressure, as follows. At low pressure, since solvent power is low, most of the primary products from coal decomposition remained in residual coal particles and thus some of these species decomposed into volatiles and others polymerized with one another. At high pressure, since solvent power is high, a large amount of primary products was dissolved in supercritical fluid and carried away from the reactor before further decomposition could occur. The slight increase in conversion with increasing pressure can be explained by the inhibition of polymerization of the primary products by rapid extraction.

The above mechanism is supported by the following two results. Both the conversion and the liquid yield at low pressure shown in Fig. 5 correspond to those at the residence time of 1.5 hour shown in Fig. 4. The conversion and the liquid yield obtained under nitrogen atmosphere were of the same level as those under low-pressure toluene atmosphere. This is because at low pressure or under nitrogen atmosphere most of the primary products remained in coal particles during the 1.5-hour experiment and thus decomposed further.

\subsection{Supercritical toluene-tetralin extraction}

The effect of tetralin addition to the supercritical toluene on the conversion and the liquid yield was examined at various pressures. In this series of experiments the residue was washed by supercritical toluene at $653 \mathrm{~K}$ and $20 \mathrm{MPa}$ after supercritical toluene-tetralin extraction to compare the results with those of supercritical toluene extraction. The results are shown in Fig. 6.

Conversion was higher than that in the case of toluene extraction even at low pressure, where the solvent power of supercritical toluene-tetralin mixture is as low as that of supercritical toluene. The higher conversion may be attributed to the inhibition of polymerization of unstable species in coal particles by the stabilization of radicals through the hydrogen transfer reaction.

Volatile yield tends to be relatively lower than that of toluene extraction, especially at lower pressure, although the accuracy of the data is not high enough. The result of supercritical toluene extraction suggests that some of the volatile species are produced by decomposition of unstable primary products. The reduced volatile yield by the addition of tetralin may be related to radical stabilization through hydrogen transfer. We think that decomposition of the primary products may be suppressed by stabilization.

The above results indicate that tetralin stabilizes

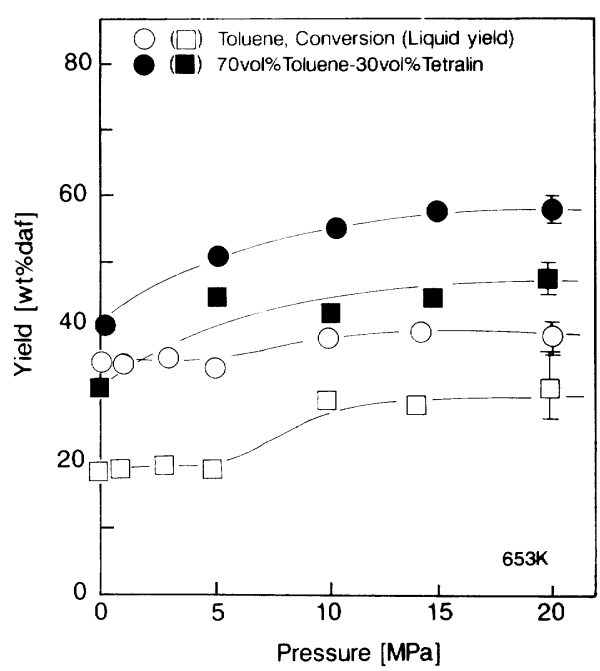

Fig. 6. Effect of pressure on conversion and liquid yield through supercritical toluene-tetralin extraction

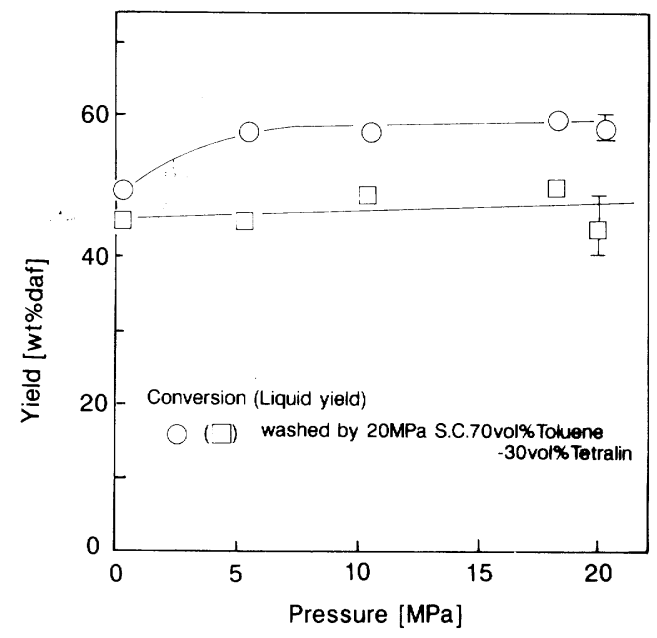

Fig. 7. Effect of pressure on conversion and liquid yield through supercritical toluene-tetralin extraction

unstable radicals even under low pressure. To examine the effect of pressure on radical stabilization, supercritical toluene-tetralin extraction was conducted at various pressures and product distributions were compared. In all the runs, the residual species in the coal bed was further recovered by supercritical toluene-tetralin mixture at $653 \mathrm{~K}$ and $20 \mathrm{MPa}$ in order to keep the product recovery conditions the same. The results are shown in Fig. 7. The conversion and liquid yield were almost constant over a wide range of pressure. Thus the results indicate that sufficient radical stabilization takes place, even at low pressure.

The effect of residence time on radical stabilization was also investigated for the toluene-tetralin extraction by comparing product distributions. As shown in Fig. 8, the volatile yield and conversion changed little over a wide range of residence time. This result suggests that unstable radicals in the coal are fully 


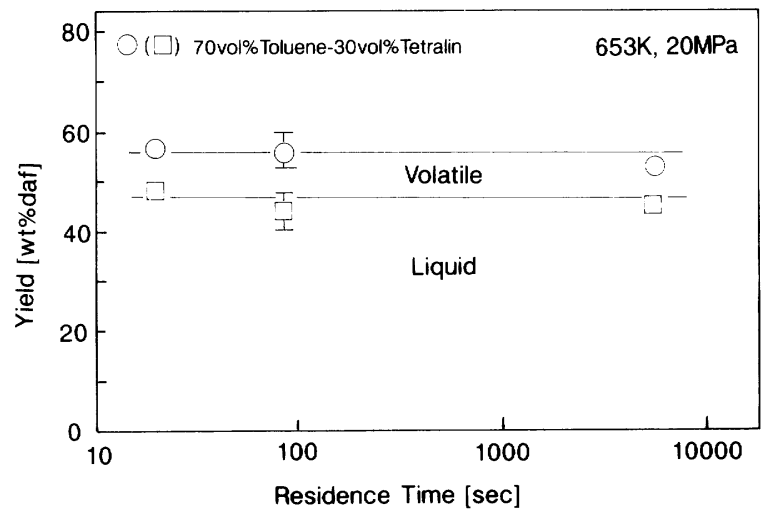

Fig. 8. Effect of residence time on conversion and liquid yield through supercritical toluene-tetralin extraction

stabilized by the hydrogen transfer reaction and are rarely decomposed or polymerize under the present conditions.

To confirm the mechanism of radical stabilization, in situ ESR measurement was conducted for supercritical toluene-tetralin extraction. ESR spectra during toluene-tetralin extraction is also shown in Fig. 3. By the addition of tetralin, the intensity decreased with increasing temperature. This result can be explained by the decrease in amount of radicals in the cell as follows. A part of the products was removed with fluid from the cell, some of the radicals in the cell were stabilized by the hydrogen transfer reaction, and stabilized species perhaps rarely decomposed into smaller radicals.

\subsection{Reaction mechanism}

One of the specific features of the mechanism of supercritical fluid extraction of coal is the effect of solvent power on the reactions during extraction. Reaction mechanisms deduced from the results obtained are shown in Fig. 9. This figure shows schematically the effect of solvent power on the radicals but does not refer to the size and amount of the species.

Various species are produced through thermal decomposition of coal. Some of these species are unstable and thus a part of them is rapidly polymerized to char and others are further decomposed into volatile matter. Supercritical toluene dissolves a part of the unstable species. Polymerization of products in coal particles is suppressed by the dissolution of these species in the solvent, although the effect is not significant for supercritical toluene extraction under the present experimental condition. When the residence time is short and solvent power is high, these unstable species are recovered as liquid products before their further decomposition. In that case, high liquid yield and low volatile yield are obtained.

Tetralin stabilizes radicals through a hydrogen transfer reaction; this prevents polymerization of

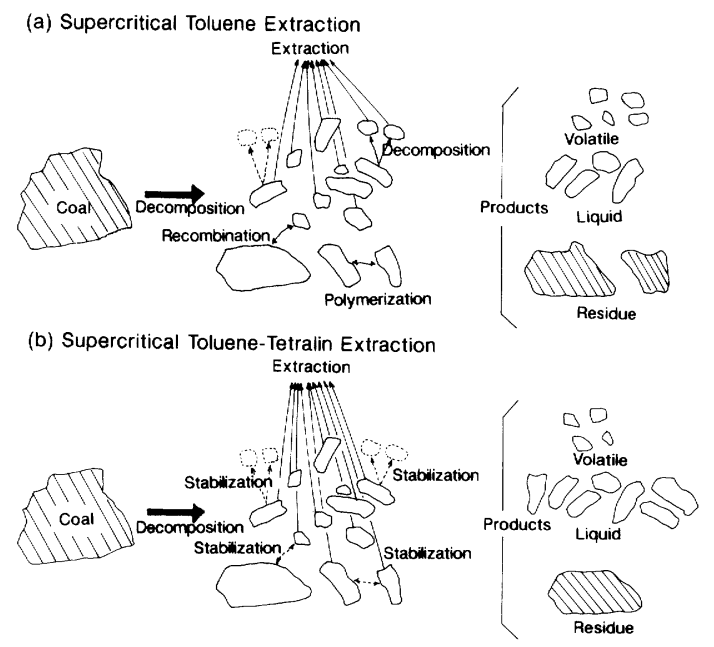

Fig. 9. Mechanism of supercritical fluid extraction of coal

primary products. Further decomposition of primary products may also be inhibited by the stabilization. This radical stabilization effectively takes place even at low pressure. By using tetralin, therefore, high conversion, high liquid yield and low volatile yield are obtained

Although this mechanism was deduced from the experimental results obtained for supercritical toluene (toluene-tetralin mixture) extraction, the same explanation can also apply to supercritical fluid extraction with other non-reactive solvents including propane, hexane, decane, and propanol, or with other hydrogen-doner solvents.

\section{Conclusion}

The effects of residence time, pressure and tetralin on the conversion and liquid yield of supercritical toluene (toluene-tetralin mixture) extraction were examined. In situ ESR measurements were also conducted to examine the change of radical concentration during the extraction. Through these detailed analyses, the effect of the solvent power of supercritical fluid (supercritical toluene and a toluene-tetralin mixture) on the reactions during extraction of coal was examined.

Conversion of coal for supercritical toluene extraction was rarely affected by the solvent power of the supercritical fluid or the residence time of the solvent under the present experimental conditions. Increased volatile yield with increasing residence time was explained by the decomposition of unstable primary products. At high pressure and short residence time, these unstable primary products were recovered as liquid products because of their rapid extraction. By the addition of tetralin, conversion and liquid yield increased while volatile yield decreased. This is because the polymerization of radicals is inhibited by the stabilization of radicals through hydrogen 
transfer from tetralin. The decrease in volatile yield may be attributed to the inhibition of decomposition of primary products by the radical stabilization.

\section{Acknowledgement}

The authors wish to express their thanks for Grants-in-aid for General Scientific Research $(01603012,02203112)$ of the Ministry of Education, Science and Culture, Japan and to Government Industrial Research, Tohoku for kindly lending us an ESR apparatus.

\section{Nomenclature}

$$
=\text { intensity of ESR spectrum }
$$

$=$ intensity of reference ESR spectrum
$X=$ conversion of coal $[\mathrm{g} / \mathrm{g}$-initial carbon in fed coal $]$

$Y_{L} \quad=$ liquid yield $\quad[\mathrm{g} / \mathrm{g}$-initial carbon in fed coal $]$

$Y_{V} \quad=$ volatile yield $\quad[\mathrm{g} / \mathrm{g}$-initial carbon in fed coal $]$

$\begin{array}{lll}W_{L} & =\text { weight } \text { of liquid } \quad \text { [g, d.a.f.] }\end{array}$

$W_{R} \quad=$ weight of residual coal $\quad$ [g, d.a.f.]

$W_{S} \quad=$ weight of sample coal [g, d.a.f.]

\section{Literature Cited}

1) Shishido, M., H. Inomata, K. Arai and S. Saito: Nenryo Kyokai-shi, 66 (3), 198 (1987).

2) Shishido, M., T. Mashiko and K. Arai: Fuel, 70, 545 (1991).

3) Shishido, M.: Dr. Thesis of Tohoku University (1989).

4) Sunol, A. K. and G. H. Beyer: Ind. Eng. Chem. Res., 29, 842 (1990).

42

